\title{
RATIONAL STREAMS COALGEBRAICALLY
}

\author{
JAN RUTTEN
}

CWI and VUA, Kruislaan 413, 1098 SJ Amsterdam

e-mail address: janr@cwi.nl

\begin{abstract}
We study rational streams (over a field) from a coalgebraic perspective. Exploiting the finality of the set of streams, we present an elementary and uniform proof of the equivalence of four notions of representability of rational streams: by finite dimensional linear systems; by finite stream circuits; by finite weighted stream automata; and by finite dimensional subsystems of the set of streams.
\end{abstract}

\section{INTRODUCTION}

A stream over a given set $A$ is an infinite sequence of elements of $A$. Streams are abound in both mathematics and computer science. Think of limits in mathematics, typically defined in terms of converging sequences, and of Taylor series of analytical functions. In computer science, streams occur in various fields such as data flow, infinite data types, semantics, formal power series, and functional programming.

In this paper, we study rational streams (over a field). They are well-known in mathematics, notably system theory, but have not received much attention in computer science. In contrast, a basic ingredient in any introductory course in the theory of computation is the notion of rational language (also called regular language). Rational languages are a prototypical example of a finitely presentable data type: a language is rational if and only if it is recognisable by a finite automaton.

As we shall see, rational streams are similarly finitely representable, in various ways. More specifically, a stream is rational iff it satisfies one of the following equivalent conditions:

(a) it is representable by a finite dimensional linear system;

(b) it is computable by a finite stream circuit;

(c) it is representable by a finite weighted stream automaton.

We shall explain the details of all of this as we will go along, but for now it is worthwhile pointing out that condition (c) is similar, in the case of languages, to being recognisable by a finite automaton. Condition (b) is particularly nice and relevant for computer scientists, since it provides a very elementary characterisation of rational streams in terms of finite memory (registers) and feedback.

1998 ACM Subject Classification: F.1.1, G.1.0.

Key words and phrases: streams, coalgebra, coinduction, linear systems, rationality, weighted automata.

This research is partially supported by EU project IST-33826 CREDO (http://credo.cwi.nl). 
Streams are for the theory of coalgebra, one could say, what the natural numbers are for algebra: a canonical example illustrating some of the essential notions of the theory. The set of natural numbers is an initial algebra (and satisfies a principle of induction). Dually, the set of streams is a final coalgebra (and satisfies a principle of coinduction). In the present paper, the proofs of the equivalence of the above three conditions will in essence be based on the finality of the set of streams. Finality moreover provides yet another equivalent characterisation of rationality. A stream is rational if and only if

(d) it generates a finite dimensional subsystem of the set of all streams.

As we shall see, this criterion is particularly useful for proving that a stream is not rational.

Most of the above and of the contents of this paper is already known, but often at different places in the literature and typically formulated in different languages, as it were. The equivalence of rationality and condition (a) above is taken as a definition in the theory of formal power series BR88. The equivalence of rationality and condition (b) is proved in system theory and in signal processing, where stream circuits are known under various names (such as signal flow graphs) [Kai80, Lah98]; see also our earlier paper [Rut07]. The equivalence between (a) and (c) is known in automata theory. Condition (d) occurs in some of our own work Rut05b; its use here to disprove rationality of a stream seems to be new.

All in all, our main goal has been to present rational streams and all of their characterisations in one place and in elementary and uniform terms. Moreover, this paper is intended as a form of publicity for rational streams to the computer science community. They provide a basic and simple model of finite memory and feedback and deserve, therewith, a place in the heart of the foundations of the theory of computing. Finally, our treatment of rational streams serves as a good illustration of the relevance of the combined use of both algebraic and coalgebraic methods in computer science.

\section{RATiOnAL STREAMS}

We define the set of streams over a given set $A$ by

$$
A^{\omega}=\{\sigma \mid \sigma:\{0,1,2, \ldots\} \rightarrow A\}
$$

We will denote elements $\sigma \in A^{\omega}$ by $\sigma=(\sigma(0), \sigma(1), \sigma(2), \ldots)$. We define the stream derivative of a stream $\sigma$ by

$$
\sigma^{\prime}=(\sigma(1), \sigma(2), \sigma(3), \ldots)
$$

and we call $\sigma(0)$ the initial value of $\sigma$. For $a \in A$ and $\sigma \in A^{\omega}$ we use the following notation:

$$
a: \sigma=(a, \sigma(0), \sigma(1), \sigma(2), \ldots)
$$

For instance, $\sigma=\sigma(0): \sigma^{\prime}$, for any $\sigma \in A^{\omega}$. (In computer science, the operations of initial value and derivative are known as head and tail.)

If the set $A$ carries some algebraic structure then typically this induces some structure on the set $A^{\omega}$ as well. In particular, if the set $A$ is a (semi-)ring

$$
A=(A,+, \cdot, 0,1)
$$

(see the Appendix for the full definition) then the set $A^{\omega}$ of streams over $A$ can be equipped with operations and constants that allow the formulation of an elementary but useful calculus. 
So let $A$ be a (semi-)ring. Examples are the set of real numbers (which is also a field) and the set of linear transformations on a vector space. We define the following operators on the set $A^{\omega}$ of streams over $A$, for all $c \in A, \sigma, \tau \in A^{\omega}, n \geq 0$ :

$$
\begin{aligned}
{[c] } & =(c, 0,0,0, \ldots) \quad(\text { often simply denoted again by } c) \\
X & =(0,1,0,0,0, \ldots) \\
(\sigma+\tau)(n) & =\sigma(n)+\tau(n) \quad[\mathrm{sum}] \\
(\sigma \times \tau)(n) & =\sum_{i=0}^{n} \sigma(i) \cdot \tau(n-i) \quad[\text { convolution product] }
\end{aligned}
$$

(where - denotes multiplication in the ring $A$ ). For the above definitions, it is already sufficient if $A$ is a semi-ring. If $A$ is moreover a ring then it comes equipped with an additive inverse, which extends to streams, for $\sigma \in A^{\omega}$, as follows:

$$
-\sigma=(-\sigma(0),-\sigma(1),-\sigma(2), \ldots)
$$

(here the minus symbols on the right are from the ring $A$ ). If the initial value $\sigma(0)$ of a stream $\sigma$ has a multiplicative inverse $\sigma(0)^{-1}$ in $A$ then $\sigma$ has a (unique) multiplicative inverse $\sigma^{-1}$ in $A^{\omega}$ :

$$
\sigma^{-1} \times \sigma=[1]
$$

As usual, we shall often write $1 / \sigma$ for $\sigma^{-1}$. If $\cdot$ and hence $\times$ is commutative, then we also write $\sigma / \tau$ for $\sigma \times \tau^{-1}$.

In general, the $n$th element $\sigma(n)$ of a stream $\sigma$ is trivially given by

$$
\sigma(n)=\sigma^{(n)}(0)
$$

where the superscript $(n)$ denotes the $n$th stream derivative. In Rut05b], various rules for the computation of stream derivatives are given. For the examples used in the present paper, all we shall be needing is the very simple rule presented in Corollary 2.5 below.

The stream operators introduced above are well behaved in that they inherit the properties of the operators of the underlying (semi-)ring. Notably sum and product are associative; sum is commutative but product $\times$ is only commutative if . is; [0] is the additive identity, [1] is the multiplicative identity. Another property we shall be using is the following. For all $\sigma \in A^{\omega}$,

$$
X \times \sigma=\sigma \times X
$$

Note that this property also holds for (semi-)rings $A$ in which multiplication is not commutative.

Since $X^{2}=(0,0,1,0,0,0, \ldots), X^{3}=(0,0,0,1,0,0,0, \ldots)$ and so on, the following infinite sum is well defined, for all $\sigma \in A^{\omega}$ :

$$
\sigma=\sigma(0)+(\sigma(1) \times X)+\left(\sigma(2) \times X^{2}\right)+\cdots
$$

(Note that we write $\sigma(i)$ for $[\sigma(i)]$; similarly below.) It shows that $\sigma$ can be viewed as a formal power series in the indeterminate $X$ (which here in fact is a constant stream). What distinguishes our approach from the classical theory of formal power series is a systematic use of the operation of stream derivative and the universal property of finality it induces (cf. Section (4). This leads to a somewhat non-standard algebraic calculus, which we call stream calculus. 
The following identity shows how one can compute a stream from its initial value and its derivative. Since this amounts to a form of (stream) integration it is called the fundamental theorem of stream calculus [Rut05b].

Theorem 2.1 (Fundamental theorem). Let $A$ be a (semi-)ring. For all $\sigma \in A^{\omega}$,

$$
\sigma=\sigma(0)+\left(X \times \sigma^{\prime}\right)
$$

Proof. Immediate from the fact that $X \times \sigma^{\prime}=(0, \sigma(1), \sigma(2), \sigma(3), \ldots)$.

For future reference, we list the following identities on initial values, which are immediate from the definition of the operations on streams.

Proposition 2.2 (Initial values). For all $\sigma \in A^{\omega}$,

$$
\begin{aligned}
(\sigma+\tau)(0) & =\sigma(0)+\tau(0) \\
(\sigma \times \tau)(0) & =\sigma(0) \cdot \tau(0) \\
\sigma^{-1}(0) & =\sigma(0)^{-1}
\end{aligned}
$$

where in the last identity $\sigma(0)$ is assumed to have a multiplicative inverse in $A$.

Next we introduce the notion of rational stream.

Definition 2.3 (Rational streams). We call a stream $\pi$ polynomial if it is of the form

$$
\begin{aligned}
\pi & =c_{0}+\left(c_{1} \times X\right)+\left(c_{2} \times X^{2}\right)+\cdots+\left(c_{k} \times X^{k}\right) \\
& =\left(c_{0}, c_{1}, c_{2}, \ldots, c_{k}, 0,0,0, \ldots\right)
\end{aligned}
$$

A stream $\rho$ is rational if it is the quotient

$$
\rho=\sigma / \tau=\sigma \times \tau^{-1}
$$

of two polynomial streams $\sigma$ and $\tau$ for which $\tau(0)^{-1}$ exists. We denote the set of all rational streams over $A$ by

$$
\operatorname{Rat}\left(A^{\omega}\right)=\left\{\sigma \in A^{\omega} \mid \sigma \text { is rational }\right\}
$$

Remark 2.4. In the literature, one also encounters the notion of rational stream defined as being ultimately periodic. In the present setting, these streams can be simply characterized as having only finitely many distinct derivatives. As we shall see in Section 5 , it follows from this basic observation that ultimately periodic streams are a special case of rational streams in our sense.

Theorem 2.1 (together with Proposition 2.2) gives an easy calculation rule for the computation of stream derivatives. First note that for all $\sigma \in A^{\omega}$,

$$
(X \times \sigma)^{\prime}=\sigma
$$

Furthermore we have, for any $\sigma \in A^{\omega}$, that $X \times \sigma^{\prime}=\sigma-\sigma(0)$, by Theorem 2.1, and $\left(X \times \sigma^{\prime}\right)^{\prime}=\sigma^{\prime}$, by (2.2). As a consequence, we have the following.

Corollary 2.5. For all $\sigma \in A^{\omega}$,

$$
\sigma^{\prime}=(\sigma-\sigma(0))^{\prime}
$$


This trivial identity makes the computation of stream derivatives often surprisingly simple.

Example 2.6. Let

with $c \in A$. We compute

$$
\sigma=\frac{1}{1-(c \times X)}
$$

$$
\begin{aligned}
\sigma^{\prime} & =(\sigma-\sigma(0))^{\prime} \quad[\text { Corollary 2.5] } \\
& =\left(\frac{1}{1-(c \times X)}-1\right)^{\prime} \quad[\text { Proposition 2.2] } \\
& =\left(\frac{c \times X}{1-(c \times X)}\right)^{\prime} \\
& =\left(X \times \frac{c}{1-(c \times X)}\right)^{\prime} \quad[\text { identity (2.1)] } \\
& =\frac{c}{1-(c \times X)} \quad[\text { identity (2.2) }]
\end{aligned}
$$

and, more generally,

$$
\sigma^{(n)}=\frac{c^{n}}{1-(c \times X)}
$$

Using the fact that $\sigma(n)=\sigma^{(n)}(0)$, this yields the following well-known expression for this prototypical example of a rational stream:

$$
\frac{1}{1-(c \times X)}=\left(1, c, c^{2}, \ldots\right)
$$

Similarly for

one computes

$$
\tau=\frac{1}{(1-X)^{2}}
$$

$$
\begin{aligned}
\tau^{\prime} & =(\tau-\tau(0))^{\prime} \\
& =\left(\frac{1}{(1-X)^{2}}-1\right)^{\prime} \\
& =\left(\frac{2 X-X^{2}}{(1-X)^{2}}\right)^{\prime} \\
& =\left(X \times \frac{2-X}{(1-X)^{2}}\right)^{\prime} \\
& =\frac{2-X}{(1-X)^{2}}
\end{aligned}
$$

and again more generally,

leading to

$$
\tau^{(n)}=\frac{(n+1)-(n \times X)}{(1-X)^{2}}
$$

$$
\frac{1}{(1-X)^{2}}=(1,2,3, \ldots)
$$




\section{StREAMS AND VECTOR SPACES}

Let $V$ be a vector space (over a field $k$ ). The set

$$
V^{\omega}=\{\sigma \mid \sigma:\{0,1,2, \ldots\} \rightarrow V\}
$$

of streams over $V$ is itself a vector space, with addition and scalar multiplication given, for $n \geq 0$ and $c \in k$, by

$$
(\sigma+\tau)(n)=\sigma(n)+\tau(n) \quad(c \cdot \sigma)(n)=c \cdot \sigma(n)
$$

where on the right we use vector addition and scalar multiplication in the vector space $V$.

For future reference, we denote the operations of initial value and derivative by $i: V^{\omega} \rightarrow V$ and $d: V^{\omega} \rightarrow V^{\omega}$ : for all $\sigma \in V^{\omega}$,

$$
i(\sigma)=\sigma(0) \quad d(\sigma)=\sigma^{\prime}
$$

Proposition 3.1. The operations of initial value $i: V^{\omega} \rightarrow V$ and derivative $d: V^{\omega} \rightarrow V^{\omega}$ are linear.

Proof. Immediate from

$$
(x \cdot \sigma+y \cdot \tau)(0)=x \cdot \sigma(0)+y \cdot \tau(0) \quad(x \cdot \sigma+y \cdot \tau)^{\prime}=x \cdot \sigma^{\prime}+y \cdot \tau^{\prime}
$$

for all $x, y \in k$ and $\sigma, \tau \in V^{\omega}$.

3.1. Streams of linear transformations. Next we define streams of linear transformations. To this end, we first note that the set

$$
L(V, V)=\{F: V \rightarrow V \mid F \text { is a linear transformation }\}
$$

(which we shall usually denote by $L$ ) is a ring

$$
\left(L,+{ }_{L},{ }_{L}, 0_{L}, 1_{L}\right)
$$

Addition $F+{ }_{L} G$, multiplication $F \cdot{ }_{L} G$, and negation $-{ }_{L} F$ are defined, for all $F, G \in L$ and $v \in V$, by

$$
\begin{aligned}
\left(F+{ }_{L} G\right)(v) & =F(v)+G(v) \\
\left(F \cdot{ }_{L} G\right)(v) & =F \circ G(v) \\
\left(-{ }_{L} F\right)(v) & =-F(v)
\end{aligned}
$$

The neutral elements $0_{L}: V \rightarrow V$ and $1_{L}: V \rightarrow V$ for sum and multiplication are given, for all $v \in V$, by $0_{L}(v)=0_{V}$ (the zero vector in $V$ ) and $1_{L}(v)=v$.

The set $L$ of linear transformations on a vector space $V$ being a ring, we have, by the definitions of Section 2, a calculus of streams of linear transformations. Streams $\phi \in L^{\omega}$ are infinite sequences $\phi=(\phi(0), \phi(1), \phi(2), \ldots)$ of linear transformations $\phi(i): V \rightarrow V$. The operations of sum and (convolution) product are given, for $\phi, \psi \in L^{\omega}$, by

$$
\begin{aligned}
(\phi+\psi)(n) & =\phi(n)+{ }_{L} \psi(n) \\
(\phi \times \psi)(n) & =\sum_{i=0}^{n} \phi(i) \cdot{ }_{L} \psi(n-i) \\
& =\sum_{i=0}^{n} \phi(i) \circ \psi(n-i)
\end{aligned}
$$


As before we also have, for every $F \in L$, a constant stream

$$
[F]=\left(F, 0_{L}, 0_{L}, 0_{L}, \ldots\right)
$$

In particular we also have

$$
\left[1_{L}\right]=\left(1_{L}, 0_{L}, 0_{L}, 0_{L}, \ldots\right)
$$

which we shall often simply denote by 1 . The constant stream $X$ now looks like

$$
X=\left(0_{L}, 1_{L}, 0_{L}, 0_{L}, 0_{L}, \ldots\right)
$$

Every stream $\phi \in L^{\omega}$ has an additive inverse $-\phi$ given, as before, by

$$
-\phi=(-\phi(0),-\phi(1),-\phi(2), \ldots)
$$

A stream $\phi \in L^{\omega}$ has a (unique) multiplicative inverse $\phi^{-1}$ in $L^{\omega}$ :

$$
\phi^{-1} \times \phi=1 \quad\left(=\left[1_{L}\right]\right)
$$

whenever the linear transformation $\phi(0): V \rightarrow V$ has a multiplicative inverse in the ring $L$, that is, whenever $\phi(0)$ is invertible.

Example 3.2. For any linear transformation $F \in L$, we define the stream $\tilde{F} \in L^{\omega}$ by

$$
\tilde{F}=\frac{1}{1-([F] \times X)}
$$

As before, it is a prototypical example of a rational stream. Note that

$$
1-([F] \times X)=\left(1_{L},-F, 0_{L}, 0_{L}, 0_{L}, \ldots\right)
$$

indeed is invertible in $L^{\omega}$ as $1_{L}$ is trivially invertible in $L$. As an instance of identity (2.3) in Example 2.6, we have

$$
\tilde{F}=\frac{1}{1-([F] \times X)}=\left(1, F, F^{2}, \cdots\right)
$$

where now $F^{n+1}=F \circ F^{n}$, all $n \geq 0$.

So far we have looked at the set $L=L(V, V)$ of streams of linear transformations from a vector spave $V$ to itself. It will also be convenient to consider (streams of) linear transformations between two different vector spaces $V$ and $W$ :

$$
L(V, W)=\{F: V \rightarrow W \mid F \text { is a linear transformation }\}
$$

Note that $L(V, W)$ is not a (semi-)ring - we cannot define multiplication to be composition as we did with $L(V, V)$ - and as a consequence the set $L(V, W)^{\omega}$ of streams over $L(V, W)$ does not have as much structure as the set $L(V, V)^{\omega}$. It will be convenient, however, to use the following generalised version of the operation of convolution product. For vector spaces $U, V, W$ and for all $\phi \in L(U, V)^{\omega}$ and $\psi \in L(V, W)^{\omega}$ we define $\psi \times \phi \in L(U, W)^{\omega}$, for all $n \geq 0$, by

$$
(\psi \times \phi)(n)=\sum_{i=0}^{n} \psi(i) \circ \phi(n-i)
$$

One can also apply streams of linear transformations to streams of vectors, as follows. For all $\phi \in L(V, W)^{\omega}$ and $\sigma \in V^{\omega}$ we define $\phi \times \sigma \in W^{\omega}$, for all $n \geq 0$, by

$$
(\phi \times \sigma)(n)=\sum_{i=0}^{n} \phi(i)(\sigma(n-i))
$$


For a linear transformation $H: V \rightarrow W$, we put again

$$
[H]=\left(H, 0_{L}, 0_{L}, 0_{L}, \ldots\right)
$$

(where now $0_{L}$ is the everywhere zero transformation from $V$ to $W$ ). As a special case of (3.3) we have

$$
[H] \times \sigma=(H(\sigma(0)), H(\sigma(1)), H(\sigma(2)), \ldots)
$$

Note that the set of streams $L(V, V)^{\omega}$ has also its own operation of convolution product, which interacts nicely with the product defined in (3.3). For instance, for $\phi, \psi \in L(V, V)^{\omega}$ and $\sigma \in V^{\omega}$,

$$
(\phi \times \psi) \times \sigma=\phi \times(\psi \times \sigma)
$$

3.2. Streams of matrices. Since linear transformations between finite dimensional vector spaces (over a field $k$ ) correspond to matrices (with entries in $k$ ), streams of linear transformations correspond to streams of matrices. Here we show how rational streams of linear transformations correspond to matrices with rational streams (over $k$ ) as entries.

First some conventions. For any set $A$ and $n \geq 1$, we denote the elements $v \in A^{n}$ by $v=\left(v_{1}, \ldots, v_{n}\right)$. It will sometimes be convenient to switch between streams of tuples and tuples of streams. We define the transpose as follows:

$$
(-)^{T}:\left(A^{n}\right)^{\omega} \rightarrow\left(A^{\omega}\right)^{n} \quad\left(\sigma^{T}\right)_{i}(j)=(\sigma(j))_{i}
$$

This function is an isomorphism and has an inverse which we denote again by

$$
(-)^{T}:\left(A^{\omega}\right)^{n} \rightarrow\left(A^{n}\right)^{\omega}
$$

Now let $k$ be a field. A linear transformation $F: k^{n} \rightarrow k^{m}$ between finite dimensional vector spaces corresponds to an $m \times n$ matrix $M_{F}$ with values $F_{i j}$ in $k$ :

$$
F: k^{n} \rightarrow k^{m} \quad M_{F}=\left(\begin{array}{cccc}
F_{11} & F_{12} & \cdots & F_{1 n} \\
F_{21} & F_{22} & \cdots & F_{2 n} \\
\vdots & \vdots & \ddots & \vdots \\
F_{m 1} & F_{m 2} & \cdots & F_{m n}
\end{array}\right)
$$

Here and in what follows, the matrix is with respect to the standard basis

$$
(1,0, \ldots, 0), \ldots,(0, \ldots, 0,1)
$$

of $k^{n}$ and $k^{m}$. Any stream $\phi=(\phi(0), \phi(1), \phi(2), \ldots)$ of linear transformations $\phi(i): k^{n} \rightarrow k^{m}$ corresponds to a stream of matrices

$$
\left(M_{\phi(0)}, M_{\phi(1)}, M_{\phi(2)}, \ldots\right)=M_{\phi(0)}+\left(M_{\phi(1)} \times X\right)+\left(M_{\phi(2)} \times X^{2}\right)+\cdots
$$

If we consider $M_{\phi(i)} \times X^{i}$ as an $m \times n$ matrix obtained from $M_{\phi(i)}$ by multiplying each of its entries by $X^{i}$, then the infinite sum on the right can itself be viewed as an $m \times n$ matrix $M_{\phi}$ with entries in $k^{\omega}$ :

$$
\left(M_{\phi}\right)_{i j}=\left(M_{\phi(0)}\right)_{i j}+\left(\left(M_{\phi(1)}\right)_{i j} \times X\right)+\left(\left(M_{\phi(2)}\right)_{i j} \times X^{2}\right)+\cdots
$$


The correspondence between $\phi$ and $M_{\phi}$ is given by the following commutative diagram:

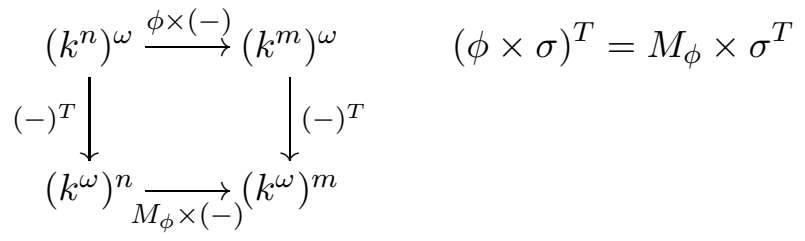

Here $\phi \times \sigma$ is as defined in (3.3) and $M_{\phi} \times \sigma^{T}$ denotes matrix to vector multiplication.

Consider $L=L\left(k^{n}, k^{n}\right)$ and recall that $\left[1_{L}\right]=\left(1_{L}, 0_{L}, 0_{L}, 0_{L}, \ldots\right)$. Let $I$ be the $n \times n$ identity matrix over $k$. We have:

$$
M_{\left[1_{L}\right]}=I
$$

Furthermore addition and convolution product of streams of linear transformations, on the one hand, and matrix addition and multiplication, on the other, are related as follows:

$$
\begin{aligned}
& M_{\phi+\psi}=M_{\phi}+M_{\psi} \\
& M_{\phi \times \psi}=M_{\phi} \times M_{\psi}
\end{aligned}
$$

As a consequence, we have the following proposition.

Proposition 3.3. Let $\rho \in L\left(k^{n}, k^{n}\right)^{\omega}$ be a stream of linear transformations $\rho(i): k^{n} \rightarrow k^{n}$. If $\rho$ is rational then $M_{\rho}$ defined in (3.6) has entries in $\operatorname{Rat}\left(k^{\omega}\right)$.

Proof: Consider two polynomial streams $\phi, \psi \in L\left(k^{n}, k^{n}\right)^{\omega}$. The entries of the matrices $M_{\phi}$ and $M_{\psi}$ are polynomial streams in $k^{\omega}$. If $\psi$ moreover has an inverse $\psi^{-1}$ then (3.8) and (3.9) imply $M_{\psi^{-1}}=\left(M_{\psi}\right)^{-1}$, which has values in $\operatorname{Rat}\left(k^{\omega}\right)$. It follows that $M_{\phi \times \psi^{-1}}=M_{\phi} \times\left(M_{\psi}\right)^{-1}$ has values in $\operatorname{Rat}\left(k^{\omega}\right)$.

Example 3.4. Let $k=\mathbb{R}$ and let $F, G: \mathbb{R}^{2} \rightarrow \mathbb{R}^{2}$ be linear transformations defined by

$$
M_{F}=\left(\begin{array}{cc}
1 & 1 \\
0 & 0
\end{array}\right) \quad M_{G}=\left(\begin{array}{cc}
0 & -1 \\
1 & 2
\end{array}\right)
$$

We compute the matrices of the rational streams $\tilde{F}=(1-(F \times X))^{-1}$ and $\tilde{G}=(1-(G \times$ $X))^{-1}$ :

$$
\begin{gathered}
M_{\tilde{F}}=\left(M_{1-(F \times X)}\right)^{-1}=\left(\begin{array}{cc}
1-X & -X \\
0 & 1
\end{array}\right)^{-1}=\left(\begin{array}{cc}
\frac{1}{1-X} & \frac{X}{1-X} \\
0 & 1
\end{array}\right) \\
M_{\tilde{G}}=\left(M_{1-(G \times X)}\right)^{-1}=\left(\begin{array}{cc}
1 & X \\
-X & 1-2 X
\end{array}\right)^{-1}=\frac{1}{(1-X)^{2}} \times\left(\begin{array}{cc}
1-2 X & -X \\
X & 1
\end{array}\right)
\end{gathered}
$$




\section{LinEAR REPRESENTATIONS}

We introduce linear systems and show how they can be used as representations for streams. In particular, we shall show how finite dimensional linear systems represent rational streams.

Let $O$ be a vector space the elements of which we think of as outputs. A linear system with output in $O$ is a pair $(V,\langle H, F\rangle)$ consisting of a vector space $V$ called the state space together with a linear transformation $F: V \rightarrow V$ called the transition function (or dynamics) and a linear transformation $H: V \rightarrow O$ called the output function.

A linear system with output in $O$ - or linear $O$-system for short — is in other words a coalgebra of the functor

$$
O \times(-): V e c t \rightarrow V e c t
$$

on the category $V e c t$ of vector spaces and linear transformations. As a consequence, there is the following (standard) notion of homomorphism. A homomorphism of linear systems $\left(V,\left\langle H_{V}, F_{V}\right\rangle\right)$ and $\left(W,\left\langle H_{W}, F_{W}\right\rangle\right)$ is a linear transformation $f: V \rightarrow W$ such that $H_{W} \circ f=$ $H_{V}$ and $F_{W} \circ f=f \circ F_{V}$ :

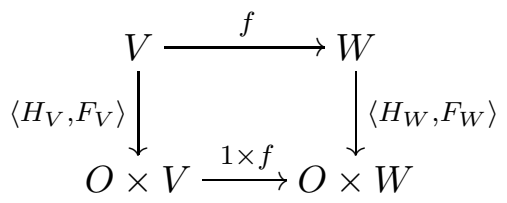

We saw (in Section 3) that if $O$ is a vector space then $O^{\omega}$ is also a vector space. Since the operations of initial value $i: O^{\omega} \rightarrow O$ and derivative $d: O^{\omega} \rightarrow O^{\omega}$ are linear transformations (Proposition 3.1), $\left(O^{\omega},\langle d, i\rangle\right)$ is a linear $O$-system. It is final among all linear $O$-systems.

Proposition 4.1 (Finality). From every linear $O$-system $(V,\langle H, F\rangle)$ there exists precisely one homomorphism to $\left(O^{\omega},\langle i, d\rangle\right)$ :

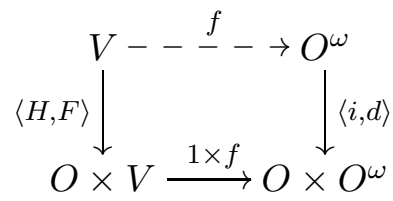

Proof. There exists precisely one function $f: V \rightarrow O^{\omega}$ making the diagram above commute. It is given by

$$
f(v)=\left(H(v), H \circ F(v), H \circ F^{2}(v), \ldots\right)
$$

for all $v \in V$ and it is linear because both $H$ and $F$ are.

Definition 4.2 (Linear representation). In the situation above, we call the stream $f(v)$ the final behaviour of $v$. We call the linear $O$-system $(V,\langle H, F\rangle)$ with designated point $v \in V$ a linear representation for the stream $\sigma \in O^{\omega}$ if $f(v)=\sigma$.

Next we look at the special case where both $O$ and $V$ are finite dimensional vector spaces over $k$. So let $n, m \geq 1$, let

$$
O=k^{m}, \quad V=k^{n}
$$

and consider a linear $k^{m}$-system $\left(k^{n},\langle H, F\rangle\right)$ with dynamics $F: k^{n} \rightarrow k^{n}$ and output $H$ : $k^{n} \rightarrow k^{m}$. The final behaviour $f: k^{n} \rightarrow\left(k^{m}\right)^{\omega}$ will map any state $v \in k^{n}$ to a stream of 
vectors in $k^{m}$. We claim that the transpose of the latter consists of a vector of $m$ rational streams in $k^{\omega}$.

Theorem 4.3. Let $n, m \geq 1$ and let $\left(k^{n},\langle H, F\rangle\right)$ be a finite dimensional $k^{m}$-system. Let

$$
f: k^{n} \rightarrow\left(k^{m}\right)^{\omega}
$$

be the final behaviour homomorphism. Then for all $v \in k^{n}$,

$$
f(v)^{T} \in \operatorname{Rat}\left(k^{\omega}\right)^{m}
$$

Proof. First we observe that for every $v \in V$, we can express $f(v)$ in terms of convolution products as follows:

$$
\begin{aligned}
f(v) & =\left(H(v), H \circ F(v), H \circ F^{2}(v), \ldots\right) \\
& =(H, 0,0,0, \ldots) \times\left(1, F, F^{2}, \ldots\right) \times(v, 0,0,0, \ldots) \quad[\text { using (3.3) and (3.4) }] \\
& =(H, 0,0,0, \ldots) \times \tilde{F} \times(v, 0,0,0, \ldots) \quad[\text { using (3.1) }] \\
& =[H] \times \tilde{F} \times[v]
\end{aligned}
$$

By (3.7), the following diagram commutes:

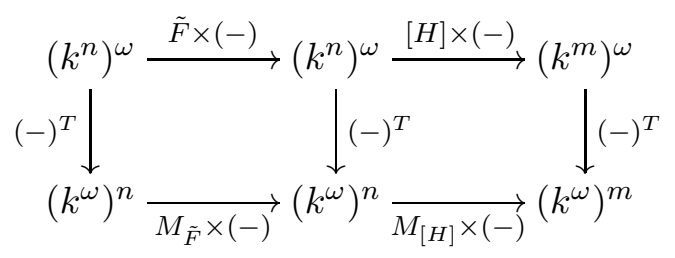

or, equivalently,

$$
([H] \times \tilde{F} \times(-))^{T}=M_{[H]} \times M_{\tilde{F}} \times(-)^{T}
$$

It follows that the final behaviour $f$ satisfies

$$
f(v)^{T}=([H] \times \tilde{F} \times[v])^{T}=M_{[H]} \times M_{\tilde{F}} \times[v]^{T}
$$

The matrix $M_{[H]}$ has entries in ( $k$ and thus in) $\operatorname{Rat}\left(k^{\omega}\right)$. Since $\tilde{F}=(1-(F \times X))^{-1}$ is a rational stream, the matrix $M_{\tilde{F}}$ has values in $\operatorname{Rat}\left(k^{\omega}\right)$, by Proposition 3.3. As a consequence, $f(v)^{T}$ is obtained from $[v]^{T}$ by multiplication with an $m \times n$ matrix with values in $\operatorname{Rat}\left(k^{\omega}\right)$. This proves the theorem.

Since finite dimensional linear systems are finitary objects (being completely determined by two finite matrices), the relevance of Theorem 4.3 lies in the fact that it shows that such finitary systems represent (vectors of) rational streams. In Section 5, we will see that any rational stream can be represented in this manner. But first we look at a few examples illustrating the present theorem.

Example 4.4. Let $k=\mathbb{R}$ and consider the linear system $\left(\mathbb{R}^{2},\langle H, F\rangle\right)$ with output $H: \mathbb{R}^{2} \rightarrow$ $\mathbb{R}$ and dynamics $F: \mathbb{R}^{2} \rightarrow \mathbb{R}^{2}$ given by

$$
H=\left(\begin{array}{ll}
1 & 1
\end{array}\right) \quad F=\left(\begin{array}{ll}
1 & 1 \\
0 & 0
\end{array}\right)
$$

The matrix $M_{\tilde{F}}$ corresponding to $\tilde{F}$ has been computed in Example 3.4

$$
M_{\tilde{F}}=\left(\begin{array}{cc}
\frac{1}{1-X} & \frac{X}{1-X} \\
0 & 1
\end{array}\right)
$$


The final behaviour $f_{\langle H, F\rangle}: \mathbb{R}^{2} \rightarrow \mathbb{R}^{\omega}$ of this system is given, for any $(a, b) \in \mathbb{R}^{2}$, by

$$
\begin{aligned}
f_{\langle H, F\rangle}(a, b) & =\left(\begin{array}{cc}
1 & 1
\end{array}\right) \times\left(\begin{array}{cc}
\frac{1}{1-X} & \frac{X}{1-X} \\
0 & 1
\end{array}\right) \times\left(\begin{array}{c}
a \\
b
\end{array}\right) \\
& =\frac{a+b}{1-X}
\end{aligned}
$$

(omitting square brackets around $a$ and $b$ as usual). Repeating the example with a different output function $\bar{H}$ and the same dynamics $F$ :

$$
\bar{H}=\left(\begin{array}{ll}
1 & 2
\end{array}\right) \quad F=\left(\begin{array}{ll}
1 & 1 \\
0 & 0
\end{array}\right)
$$

leads to the following final behaviour:

$$
f_{\langle\bar{H}, F\rangle}(a, b)=\left(\begin{array}{cc}
\frac{1}{1-X} & \frac{2-X}{1-X}
\end{array}\right) \times\left(\begin{array}{c}
a \\
b
\end{array}\right)=\frac{a+2 b-b X}{1-X}
$$

Because linear $O$-systems are coalgebras, the general definition of coalgebraic equivalence applies. In conclusion of this section, we spell out this definition together with the observation that the corresponding minimization of a system is given by (the image under) the final behaviour mapping.

Equivalence of linear $O$-systems is defined as follows. A relation $R \subseteq V \times W$ is called an $O$-bisimulation between $O$-systems $\left(V,\left\langle H_{V}, F_{V}\right\rangle\right)$ and $\left(W,\left\langle H_{W}, F_{W}\right\rangle\right)$ if for all $v \in V$ and $w \in W:$

$$
\langle v, w\rangle \in R \Rightarrow\left\{\begin{array}{c}
H_{V}(v)=H_{W}(w) \text { and } \\
\left\langle F_{V}(v), F_{W}(w)\right\rangle \in R
\end{array}\right.
$$

We say that $v$ and $w$ are $O$-equivalent and write $v \sim_{O} w$ if there exists an $O$-bisimulation $R$ with $\langle v, w\rangle \in R$. The final behaviour $f: V \rightarrow O^{\omega}$ of an $O$-system $\left(V,\left\langle H_{V}, F_{V}\right\rangle\right)$ identifies precisely all $O$-equivalent states: $v_{1} \sim_{O} v_{2}$ iff $f\left(v_{1}\right)=f\left(v_{2}\right)$, for all $v_{1}, v_{2} \in V$. (For the elementary proof, see Rut05b.) As a consequence, the minimization of an $O$-system with respect to $O$-equivalence is given by the image of $V$ under $f$, which is a subsystem $f(V) \subseteq O^{\omega}$ because $f$ is a homomorphism. It follows that the greatest $O$-equivalence on $V$ is given by the kernel $\operatorname{ker}(f)$.

\section{Constructing Linear Representations}

Let $k$ be a field. We show how to construct finite-dimensional linear representations for (vectors of) rational streams in $k^{\omega}$.

For a stream $\sigma \in O^{\omega}$ we consider the smallest subspace of $O^{\omega}$ that contains $\sigma$ and is closed under the operation of stream derivative, that is, the linear transformation $d: O^{\omega} \rightarrow O^{\omega}$. This (so-called $d$-cyclic) vector space $Z_{\sigma}$ is the subspace of $O^{\omega}$ that is spanned by the set of vectors given by

$$
\left\{\sigma^{(0)}, \sigma^{(1)}, \sigma^{(2)}, \ldots\right\}
$$

with $\sigma^{(0)}=\sigma$ and $\sigma^{(n+1)}=d\left(\sigma^{(n)}\right)=\left(\sigma^{(n)}\right)^{\prime}$. We can turn $Z_{\sigma}$ into a linear system by taking as output function and transition function the restrictions of $i: O^{\omega} \rightarrow O$ and $d: O^{\omega} \rightarrow O^{\omega}$ to $Z_{\sigma}$. The set inclusion

$$
f: Z_{\sigma} \subseteq O^{\omega}
$$


is then a homomorphism of linear $O$-systems. By finality of $\left(O^{\omega},\langle i, d\rangle\right)$, this homomorphism is unique. It follows that $\left(Z_{\sigma},\langle i, d\rangle\right)$ with initial state $\sigma$ is a minimal representation of $\sigma$.

In general, the dimension of $Z_{\sigma}$ will be infinite. Of special interest are those $\sigma \in O^{\omega}$ for which there exists an $n \geq 1$ such that all of $\sigma=\sigma^{(0)}$ through $\sigma^{(n-1)}$ are linearly independent and

$$
\sigma^{(n)}=\sum_{i=0}^{n} c_{i} \times \sigma^{(i)}
$$

for some coefficients $c_{0}, \ldots, c_{n-1}$ in the base field $k$ of $O$ and $O^{\omega}$. Then $Z_{\sigma}$ is a vector space of dimension $n$. The linear transformation $G: Z_{\sigma} \rightarrow Z_{\sigma}$ induced by $d: O^{\omega} \rightarrow O^{\omega}$ is given, with respect to the (ordered) basis $\sigma^{(0)}, \ldots, \sigma^{(n-1)}$, by the $n \times n$ matrix

$$
M_{G}=\left(\begin{array}{ccccc}
0 & 0 & \cdots & 0 & c_{0} \\
1 & 0 & \cdots & 0 & c_{1} \\
0 & 1 & \cdots & 0 & c_{2} \\
\vdots & \vdots & \ddots & \vdots & \vdots \\
0 & 0 & \cdots & 1 & c_{n-1}
\end{array}\right)
$$

(This matrix is in fact (a variation of) the companion matrix of the so-called $d$-order polynomial of $\sigma$; cf. [BM77, Thm.15, p.339].) The linear transformation $H: Z_{\sigma} \rightarrow O$ induced by $i: O^{\omega} \rightarrow O$ is given, again with respect to the basis $\sigma^{(0)}, \ldots, \sigma^{(n-1)}$, by the matrix (of size $\operatorname{dim}(O) \times n)$

$$
M_{H}=\left(\begin{array}{lllll}
\sigma^{(0)}(0) & \sigma^{(1)}(0) & \sigma^{(2)}(0) & \cdots & \sigma^{(n-1)}(0)
\end{array}\right)
$$

Thus we have obtained a linear $O$-system $\left(Z_{\sigma},\langle H, G\rangle\right)$ of dimension $n$. As before, the inclusion $f: Z_{\sigma} \subseteq O^{\omega}$ is a homomorphism. Thus $f(\tau)=\tau$, for all $\tau \in Z_{\sigma}$ and $\left(Z_{\sigma},\langle H, G\rangle\right)$ with $\sigma$ as initial state is a minimal representation of $\sigma$.

Example 5.1. Let $O=\mathbb{R}$ and consider the stream $\sigma=1 /(1-X)^{2} \in O^{\omega}$. Computing the successive stream derivatives of $\sigma=\sigma^{(0)}$, using Corollary 2.5, gives

$$
\sigma^{(1)}=\frac{2-X}{(1-X)^{2}} \quad \sigma^{(2)}=\frac{3-2 X}{(1-X)^{2}}=-\sigma^{(0)}+\left(2 \times \sigma^{(1)}\right)
$$

Thus $\sigma^{(0)}$ and $\sigma^{(1)}$ form a basis for $Z_{\sigma}$. Because $\sigma^{(0)}(0)=1$ and $\sigma^{(1)}(0)=2$, we have

$$
M_{H}=\left(\begin{array}{cc}
1 & 2
\end{array}\right) \quad M_{G}=\left(\begin{array}{cc}
0 & -1 \\
1 & 2
\end{array}\right)
$$

Now $\sigma$ is represented by $\left(Z_{\sigma},\langle H, G\rangle\right)$, with $\sigma$ as the initial state. Clearly, $\mathbb{R}^{2} \cong Z_{\sigma}$. Note that this isomorphism can also be obtained by computing the final behaviour $f: \mathbb{R}^{2} \rightarrow \mathbb{R}^{\omega}$ of the $O$-system $\left(\mathbb{R}^{2},\langle H, G\rangle\right)$, using Theorem 4.3. This gives, for all $\left(r_{1}, r_{2}\right) \in \mathbb{R}^{2}$,

$$
\begin{aligned}
f\left(r_{1}, r_{2}\right) & =M_{H} \times M_{\tilde{G}} \times\left(r_{1}, r_{2}\right) \\
& =\left(\begin{array}{ll}
1 & 2
\end{array}\right) \times \frac{1}{(1-X)^{2}} \times\left(\begin{array}{cc}
1-2 X & -X \\
X & 1
\end{array}\right) \times\left(\begin{array}{c}
r_{1} \\
r_{2}
\end{array}\right)
\end{aligned}
$$

(Recall the computation of $M_{\tilde{G}}$ from Example 3.4.) As expected, we have $f(1,0)=\sigma$ and $f(0,1)=\sigma^{(1)}$. 
Example 5.2. Let $O=\mathbb{R}^{2}$ and consider the pair $(\tau, \sigma) \in\left(\mathbb{R}^{\omega}\right)^{2} \cong\left(\mathbb{R}^{2}\right)^{\omega}$, with $\tau=$ $1 /(1-2 X)$ and $\sigma=1 /(1-X)^{2}$. Computing (pairs of) stream derivatives

$$
\begin{aligned}
(\tau, \sigma)^{(1)} & =\left(\frac{2}{1-2 X}, \frac{2-X}{(1-X)^{2}}\right) \\
(\tau, \sigma)^{(2)} & =\left(\frac{2^{2}}{1-2 X}, \frac{3-2 X}{(1-X)^{2}}\right) \\
(\tau, \sigma)^{(3)} & =\left(\frac{2^{3}}{1-2 X}, \frac{4-3 X}{(1-X)^{2}}\right) \\
& =2 \times(\tau, \sigma)^{(0)}-5 \times(\tau, \sigma)^{(1)}+4 \times(\tau, \sigma)^{(2)}
\end{aligned}
$$

we see that $Z_{(\tau, \sigma)}$ has dimension 3 with $H: Z_{(\tau, \sigma)} \rightarrow \mathbb{R}^{2}$ and $G: Z_{(\tau, \sigma)} \rightarrow Z_{(\tau, \sigma)}$ given by

$$
M_{H}=\left(\begin{array}{ccc}
1 & 2 & 4 \\
1 & 2 & 3
\end{array}\right) \quad M_{G}=\left(\begin{array}{ccc}
0 & 0 & 2 \\
1 & 0 & -5 \\
0 & 1 & 4
\end{array}\right)
$$

Theorem 5.3. Let $k$ be a field and let $O=k^{m}$. A vector of streams $\sigma \in\left(k^{\omega}\right)^{m}$ is representable by a linear $k^{m}$-system of finite dimension iff $\sigma \in\left(\operatorname{Rat}\left(k^{\omega}\right)\right)^{m}$.

Proof. From left to right, this is Theorem 4.3. For the converse, it is sufficient to observe that the examples above generalise to arbitrary vectors of rational streams. This is immediate from the fact that for a rational stream $\sigma=\rho / \tau$, the dimension of $Z_{\sigma}$ in the construction above is bounded by the maximum of the degrees of $\rho$ and $\tau$.

For single streams, the results of this section can be summarized as follows.

Theorem 5.4. Let $k$ be a field. For a stream $\sigma \in k^{\omega}$, the following are equivalent:

(1) The stream $\sigma$ is rational: $\sigma=\rho / \tau$ for polynomial streams $\rho$ and $\tau$ (with $\tau(0) \neq 0$ ).

(2) The stream $\sigma$ is representable by a linear system of finite dimension.

(3) The subsystem $Z_{\sigma} \subseteq\left(k^{\omega},\langle i, d\rangle\right)$ generated by $\sigma$ has finite dimension.

In conclusion of this section, we show that (3) above can be conveniently used to prove that a stream is not rational.

Corollary 5.5. In order to prove that a stream $\sigma \in k^{\omega}$ is not rational, it suffices to show that

$$
\left\{\sigma^{(0)}, \sigma^{(1)}, \sigma^{(2)}, \ldots\right\} \subseteq k^{\omega}
$$

contains infinitely many linearly independent vectors.

Example 5.6. Consider $\sigma \in \mathbb{R}^{\omega}$ given by

$$
\begin{aligned}
\sigma & =(1,1,0,1,0,0,1,0,0,0,1,0,0,0,0, \ldots) \\
& =1+X+X^{3}+X^{6}+X^{10}+X^{15}+\cdots \\
& =\sum_{k=0}^{\infty} X^{k(k+1) / 2}
\end{aligned}
$$


The set of stream derivatives of $\sigma$ contains the following infinite subset of linearly independent streams:

$$
\begin{gathered}
(1, \ldots) \\
(0,1, \ldots) \\
(0,0,1, \ldots) \\
(0,0,0,1, \ldots)
\end{gathered}
$$

Thus $\sigma$ is not rational.

\section{Stream Circuits}

We saw that rational streams can be represented by finite dimensional linear systems. Such systems are finitary in that they are determined by (two) finite dimensional matrices with values in $k$. In this section, we show that such systems - and as a consequence rational streams - are, equivalently, computed by so-called stream circuits with finite memory.

Stream circuits (with values in a field $k$ ) are data flow networks that act on streams of inputs and produce streams of outputs. They are built out of four types of basic gates by means of composition, which amounts simply to connecting (single) output ends to (single) input ends. We introduce these basic gates below, first describing their single-step behaviour in terms of input and output values (in $k$ ). Next we shall describe their behaviour in terms of input and output streams (in $k^{\omega}$ ).

(i) For a fixed $r \in k$, an $r$-multiplier

$$
x \longmapsto r \cdot x
$$

inputs a value $x \in k$ at its input end and outputs that value multiplied with $r$ at its output end.

(ii) A register

$$
y \longmapsto x
$$

is a one-element buffer (or memory cell) containing as initial value an element $x \in k$. Its stepwise computation consists of the (simultaneous) output of the present value $x$ in the buffer together with the input of an element $y \in k$, which becomes the new contents of the buffer:

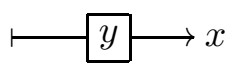

(iii) An adder

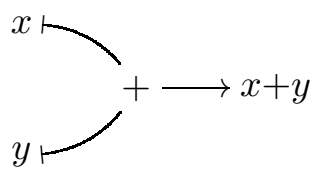

takes two input values at its input ends and outputs their sum at its output end. Here we show a 2 -to- 1 adder but more generally we will also use $n$-to- 1 adders, for $n \geq 2$. 
(iv) Lastly a copier

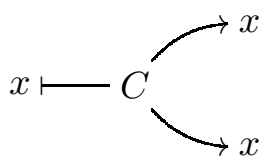

inputs a value at its input end and outputs multiple copies of it at its output ends. Here we show a 1-to- 2 copier but more generally we will also use 1-to- $n$ copiers, for $n \geq 2$.

Sometimes it will be convenient to combine multipliers with adders (and similarly copiers). For instance,

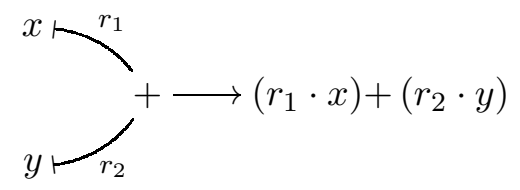

multiplies its inputs $x$ and $y$ with the values $r_{1}$ and $r_{2}$ and outputs the sum of the results.

The presence of memory (in the form of registers) makes that the behaviour of stream circuits cannot be described simply in terms of functions of single input and output values in $k$. (This is reflected in our explanations above by the fact that we needed two pictures to illustrate the behaviour of a register.) Rather we shall describe the behaviour of our circuits in terms of streams of inputs and outputs. As it turns out, all we need are the basic operations of stream calculus:

(i) An $r$-multiplier converts a stream of inputs $\sigma \in k^{\omega}$

$$
\sigma \longmapsto[r] \times \sigma
$$

into a stream of outputs $[r] \times \sigma$ by elementwise multiplying the input values with $r$ :

$$
[r] \times \sigma=(r \cdot \sigma(0), r \cdot \sigma(1), r \cdot \sigma(2), \ldots)
$$

(ii) A register with initial value $x \in k$ takes a stream of inputs $\sigma$

$$
\sigma \longmapsto x \longrightarrow[x]+(X \times \sigma)
$$

and outputs it with one step delay, after having output the initial value $x$ first:

$$
[x]+(X \times \sigma)=(x, \sigma(0), \sigma(1), \ldots)
$$

(iii) An adder takes two input streams $\sigma$ and $\tau$

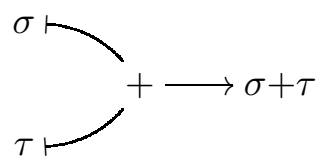

and outputs the stream consisting of their elementwise addition:

$$
\sigma+\tau=(\sigma(0)+\tau(0), \sigma(1)+\tau(1), \sigma(2)+\tau(2), \ldots)
$$

(iv) The copier simply copies input streams into output streams:

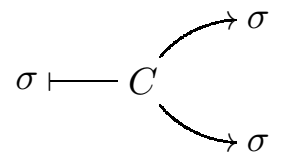


Combinations of multipliers and adders (and similarly copiers) have the expected stream behaviour:

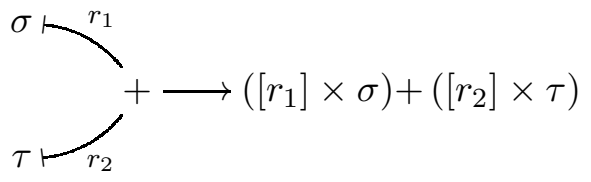

Now that we have seen the basic gates and their behaviour, let us look at composite stream circuits and see how their behaviour can be computed from that of the gates from which they are made. Consider the following circuit, built out of two registers, two copiers, three adders, and six multipliers (two of which are combined with the adder at the bottom):

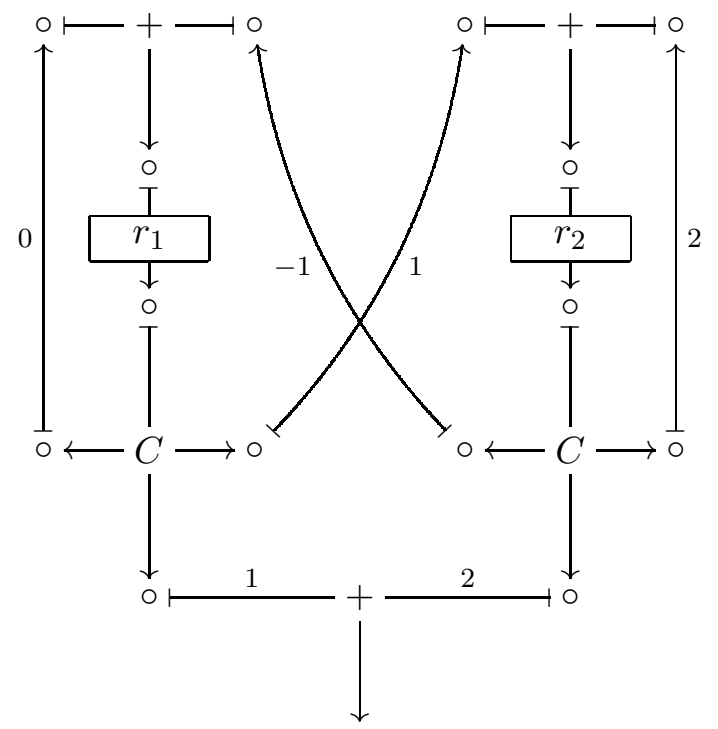

In the picture above, we use $\circ$ to denote the composition of an output end with an input end. The circuit as a whole has no external input ends and one external output end. The heart of the circuit consists of two registers with initial values $r_{1}$ and $r_{2}$. The outputs of the registers are copied and:

(a) fed back to the input ends of the registers, via multipliers whose values can be expressed by the following $2 \times 2$ matrix:

$$
M=\left(\begin{array}{cc}
0 & -1 \\
1 & 2
\end{array}\right)
$$

This leads to new values of the registers given by

$$
\left(\begin{array}{cc}
0 & -1 \\
1 & 2
\end{array}\right)\left(\begin{array}{c}
r_{1} \\
r_{2}
\end{array}\right)=\left(\begin{array}{c}
-r_{2} \\
r_{1}+2 r_{2}
\end{array}\right)
$$

(b) At the same time, the outputs of the registers are fed forward into an adder combined with multipliers whose values are given by the following matrix:

$$
N=\left(\begin{array}{ll}
1 & 2
\end{array}\right)
$$

This leads to a (first) output value given by

$$
\left(\begin{array}{ll}
1 & 2
\end{array}\right)\left(\begin{array}{l}
r_{1} \\
r_{2}
\end{array}\right)=r_{1}+2 r_{2}
$$


We call this a circuit in canonical form. More generally, we have the following definition.

Definition 6.1. We say that a stream circuit is in canonical form if it has no input ends and one output end; consists of $n \geq 1$ registers with feedback lines given by an $n \times n$ matrix; and has feedforward lines given by an $1 \times n$ matrix leading via an $n$-to- 1 adder to a single output end.

In the description of the example circuit above, (a) and (b) together describe one single atomic computation step of the circuit. Next we describe the stream behaviour of our example canonical circuit. As we shall see, the output end of a canonical stream circuit produces precisely one (rational) stream. In order to compute this output stream, we first give names ( $\sigma$ and $\tau$ ) to the streams that will occur at the output ends of the two registers. Then we apply the stream equations for each of the basic gates in the circuit, leading to:

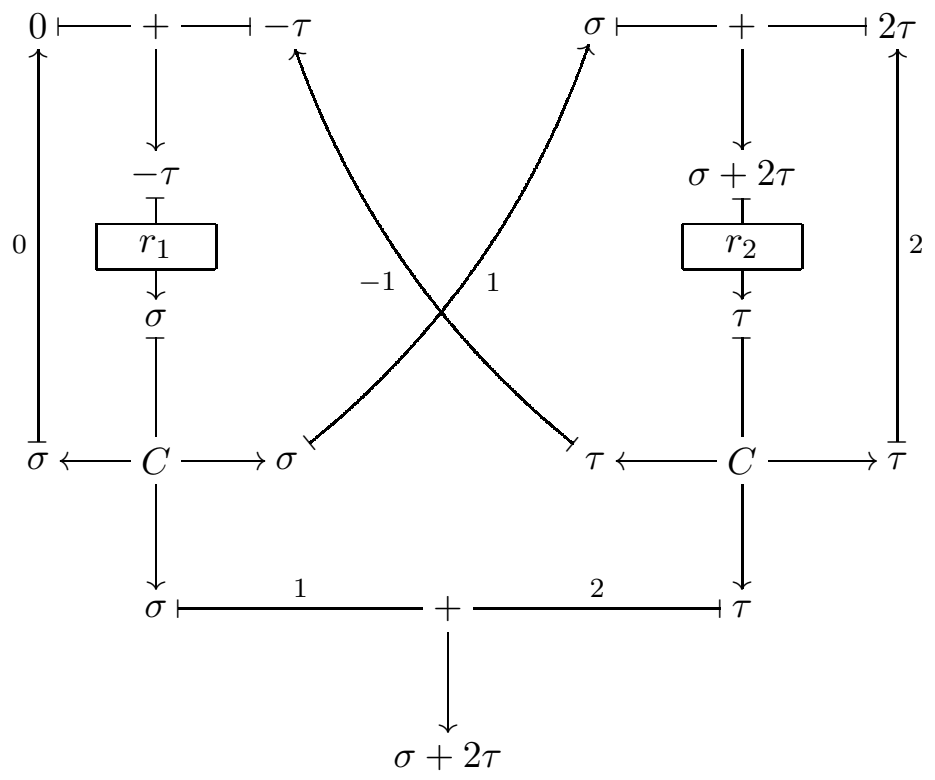

Applying the register law (6.1) to our two registers then leads to the following two equations (writing $r$ for $[r]$ as usual):

$$
\begin{aligned}
\sigma & =r_{1}+(X \times-\tau) \\
\tau & =r_{2}+(X \times(\sigma+2 \tau))
\end{aligned}
$$

or, equivalently, in matrix notation:

$$
\left(\begin{array}{l}
\sigma \\
\tau
\end{array}\right)=\left(\begin{array}{l}
r_{1} \\
r_{2}
\end{array}\right)+X \times\left(\begin{array}{cc}
0 & -1 \\
1 & 2
\end{array}\right) \times\left(\begin{array}{l}
\sigma \\
\tau
\end{array}\right)
$$

whence

$$
\left(\begin{array}{cc}
1 & X \\
-X & 1-2 X
\end{array}\right) \times\left(\begin{array}{l}
\sigma \\
\tau
\end{array}\right)=\left(\begin{array}{l}
r_{1} \\
r_{2}
\end{array}\right)
$$

This leads to the following values for $\sigma$ and $\tau$ :

$$
\left(\begin{array}{l}
\sigma \\
\tau
\end{array}\right)=\left(\begin{array}{cc}
1 & X \\
-X & 1-2 X
\end{array}\right)^{-1} \times\left(\begin{array}{l}
r_{1} \\
r_{2}
\end{array}\right)
$$


(recall that this inverse matrix was computed in Example 3.4). As a consequence the output stream of the circuit is given by

$$
\sigma+2 \tau=\left(\begin{array}{ll}
1 & 2
\end{array}\right) \times\left(\begin{array}{cc}
1 & X \\
-X & 1-2 X
\end{array}\right)^{-1} \times\left(\begin{array}{l}
r_{1} \\
r_{2}
\end{array}\right)
$$

We saw that the above circuit is fully determined by the two matrices $M$ and $N$ containing the values of the (feedback and feedforward) multipliers. As such, the circuit corresponds precisely to a linear system $\left(k^{2},\langle H, G\rangle\right)$ with $G: k^{2} \rightarrow k^{2}$ and $H: k^{2} \rightarrow k$ given by

$$
G\left(r_{1}, r_{2}\right)=M \times\left(\begin{array}{c}
r_{1} \\
r_{2}
\end{array}\right) \quad H\left(r_{1}, r_{2}\right)=N \times\left(\begin{array}{c}
r_{1} \\
r_{2}
\end{array}\right)
$$

A state of this linear system corresponds to the contents of the two registers of the circuit; $G\left(r_{1}, r_{2}\right)$ corresponds to the feedback multiplication with the matrix $M$; and the output given by $H\left(r_{1}, r_{2}\right)$ corresponds to the feed-forward multiplication with the matrix $N$. Note that the stream behaviour of our circuit as described above corresponds precisely with the (final) behaviour of the corresponding linear systems, as given by (the proof of) Theorem 4.3 in Section 4. This follows from the fact that identity (6.2) equals

$$
\begin{aligned}
\sigma+2 \tau & =\left(\begin{array}{ll}
1 & 2
\end{array}\right) \times\left(\begin{array}{cc}
1 & X \\
-X & 1-2 X
\end{array}\right)^{-1} \times\left(\begin{array}{l}
r_{1} \\
r_{2}
\end{array}\right) \\
& =M_{[H]} \times M_{\tilde{G}} \times\left(\begin{array}{c}
r_{1} \\
r_{2}
\end{array}\right)
\end{aligned}
$$

with $H$ and $G$ as defined above.

Summarizing, we have presented an example of a canonical stream circuit and shown that how to compute the (rational) stream that it produces at its output end. Then we observed that such a canonical stream circuit corresponds precisely to a finite dimensional linear system via its two matrices of feedback and feedforward multipliers. Moreover, the stream behaviour of the circuit coincides with that of the linear system.

In conclusion of this section, we note that one can construct, conversely, from any finite dimensional linear system $(V,\langle H, G\rangle)$ a corresponding canonical stream circuit with exactly the same stream behaviour: the dimension of $V$ determines the number of registers; the matrix corresponding to $G$ determines the values of the feedback multipliers; and the matrix corresponding to $H$ determines the values of the feedforward lines.

All in all, we have proved the following.

Theorem 6.2. Let $k$ be a field. For $\sigma \in k^{\omega}$, the following are equivalent:

(1) The stream $\sigma$ is representable by a linear system of finite dimension.

(2) The stream $\sigma$ is computable by a finite stream circuit.

\section{Weighted stream automata}

We saw that rational streams are "finite memory": they can be computed by stream circuits with finitely many registers. In this section, we show they are also "finite state": they can be computed by finite so-called weighted stream automata.

A weighted stream automaton with values in a field $k$ is a pair $(Q,\langle o, t\rangle)$ consisting of a set $Q$ of states, together with an output function $o: Q \rightarrow k$ and a transition function 
$t: Q \rightarrow(Q \rightarrow k)$. The output function $o$ assigns to each state $q \in Q$ a value $o(q) \in k$ called the output of $q$. The transition function $t$ assigns to each state $q \in Q$ a function $t(q): Q \rightarrow k$, which specifies for any state $q^{\prime} \in Q$ a value $t(q)\left(q^{\prime}\right) \in k$. This number can be thought of as the weight with which the transition from $q$ to $q^{\prime}$ occurs. (There are various possible interpretations of this notion of weight, such as the cost, multiplicity, duration etc. of the transition.) We will use the following notation:

$$
q \stackrel{r}{\rightarrow} q^{\prime} \equiv t(q)\left(q^{\prime}\right)=r, \quad q \stackrel{r}{\Rightarrow} \equiv o(q)=r
$$

Weighted stream automata represent streams in $k^{\omega}$ in the following manner.

Definition 7.1. For a state $q \in Q$ of a weighted stream automaton $(Q,\langle o, t\rangle)$ we define a stream $S(q)$, for all $k \geq 0$, by

$$
\begin{aligned}
& S(q)(k)= \\
& \quad \sum\left\{l_{0} \times l_{1} \times \cdots \times l_{k-1} \times l \mid \exists q_{0}, \ldots, q_{k}: q=q_{0} \stackrel{l_{0}}{\longrightarrow} q_{1} \stackrel{l_{1}}{\longrightarrow} \cdots \stackrel{l_{k-1}}{\longrightarrow} q_{k} \stackrel{l}{\Rightarrow}\right\}
\end{aligned}
$$

$($ Here $\times$ denotes multiplication in the field $k$.)

So the $k t h$ value of the stream $S(q)$ is obtained by considering all transition paths of length $k$ starting in the state $q$; multiplying for each such path the labels of all transitions; and adding up the resulting values for all paths. We say that the stream $S(q)$ is represented by the state $q$.

Example 7.2. Consider the following example of a weighted automaton:

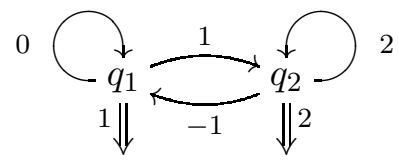

Computing the streams $S\left(q_{1}\right)$ and $S\left(q_{2}\right)$ according to Definition 7.1 above gives

$$
S\left(q_{1}\right)=(1,2,3, \ldots), \quad S\left(q_{2}\right)=(2,3,4, \ldots)
$$

We can represent all information contained in the definition of weighted stream automata by two matrices, in very much the same way as we could define stream circuits by two matrices as well. To this end, we define for a weighted stream automaton $(Q,\langle o, t\rangle)$, with states $\left\{q_{1}, \ldots, q_{n}\right\}$, an output matrix $L$ and a transition matrix $K$ as follows:

$$
L_{i}=o\left(q_{i}\right), \quad K_{i j}=t\left(q_{i}\right)\left(q_{j}\right)
$$

Now we can compute the streams represented by the states of a weighted automaton directly in terms of these matrices. Illustrating this for the example automaton above, we have

$$
L=\left(\begin{array}{l}
1 \\
2
\end{array}\right) \quad K=\left(\begin{array}{cc}
0 & 1 \\
-1 & 2
\end{array}\right)
$$

Applying Theorem 2.1 to the (vector of) streams

$$
\sigma=S\left(q_{1}\right), \quad \tau=S\left(q_{2}\right)
$$

we obtain

$$
\left(\begin{array}{c}
\sigma \\
\tau
\end{array}\right)=\left(\begin{array}{c}
\sigma(0) \\
\tau(0)
\end{array}\right)+X \times\left(\begin{array}{c}
\sigma^{\prime} \\
\tau^{\prime}
\end{array}\right)
$$


Note that it follows from Definition 7.1 that

$$
\left(\begin{array}{l}
\sigma(0) \\
\tau(0)
\end{array}\right)=L
$$

and

$$
\left(\begin{array}{l}
\sigma^{\prime} \\
\tau^{\prime}
\end{array}\right)=K \times\left(\begin{array}{l}
\sigma \\
\tau
\end{array}\right)
$$

As a consequence, we find

$$
\left(\begin{array}{l}
\sigma \\
\tau
\end{array}\right)=\left(\begin{array}{l}
1 \\
2
\end{array}\right)+X \times\left(\begin{array}{cc}
0 & 1 \\
-1 & 2
\end{array}\right) \times\left(\begin{array}{c}
\sigma \\
\tau
\end{array}\right)
$$

which leads to

$$
\begin{aligned}
\left(\begin{array}{l}
\sigma \\
\tau
\end{array}\right) & =\left(\begin{array}{cc}
1 & -X \\
X & 1-2 X
\end{array}\right)^{-1} \times\left(\begin{array}{l}
1 \\
2
\end{array}\right) \\
& =\frac{1}{(1-X)^{2}} \times\left(\begin{array}{cc}
1-2 X & X \\
-X & 1
\end{array}\right) \times\left(\begin{array}{l}
1 \\
2
\end{array}\right)
\end{aligned}
$$

It follows that

$$
S\left(q_{1}\right)=\sigma=\frac{1}{(1-X)^{2}}, \quad S\left(q_{2}\right)=\tau=\frac{2-X}{(1-X)^{2}}
$$

showing that the streams represented by our weighted automaton are rational. All of the above generalises directly to arbitrary weighted automata and so we have proved one half of the following theorem.

Theorem 7.3. A stream $\sigma \in k^{\omega}$ is rational iff it can be represented by a state $q \in Q$ of a finite weighted stream automata $(Q,\langle o, t\rangle)$ with values in $k$.

Proof. The implication from right to left follows from the above. For the converse, consider a rational stream $\sigma \in k^{\omega}$. It follows from Theorem 5.4 that $\sigma$ is representable by a linear system of finite dimension $\left(k^{n},\langle H, F\rangle\right)$ with output in $k$. Without loss of generality we can assume that $\sigma$ is represented by the vector $(1,0, \ldots, 0) \in k^{n}$. We define $Q=\left\{q_{1}, \ldots, q_{n}\right\}$ by

$$
q_{1}=(1,0, \ldots, 0), \ldots, q_{n}=(0, \ldots, 0,1)
$$

Next we define a weighted stream automaton $(Q,\langle o, t\rangle)$ by putting, for all $1 \leq i, j \leq n$,

$$
o\left(q_{i}\right)=H_{i}, \quad t\left(q_{i}\right)\left(q_{j}\right)=F_{i j}^{T}=F_{j i}
$$

It follows that $\sigma=S\left(q_{1}\right)$, that is, $\sigma$ is represented by the state $q_{1}$ in $(Q,\langle o, t\rangle)$.

\section{Summary AND DisCussion}

All in all, we have proved the following.

Theorem 8.1. Let $k$ be a field. For a stream $\sigma \in k^{\omega}$, the following are equivalent:

(1) The stream $\sigma$ is rational: $\sigma=\rho / \tau$ for polynomial streams $\rho$ and $\tau$ (with $\tau(0) \neq 0$ ).

(2) The stream $\sigma$ is representable by a linear system of finite dimension.

(3) The subsystem $Z_{\sigma} \subseteq\left(k^{\omega},\langle i, d\rangle\right)$ generated by $\sigma$ has finite dimension.

(4) The stream $\sigma$ is computable by a finite stream circuit.

(5) The stream $\sigma$ is representable by a finite weighted stream automaton. 
We mention a few examples of the many interesting questions and directions that remain to be explored. Streams over a finite field enjoy many special properties. A special example is the family of bitstreams, which consist of 0's and 1's. The interplay between coalgebraic techniques and various algebraic structures on bitstreams, such as the Boolean and the 2-adic operators, deserves further study, which may also be relevant for the construction and analysis of digital circuits; see [Rut05a, HCR06] for some preliminary results. There is also much and interesting life beyond rationality. For instance, it would be worthwhile to try and apply coinductive techniques to the study of so-called automatic sequences, see for instance [AS03. Another example is the combined use of linear systems theory and coalgebra in the world of hybrid systems, where discrete time and continuous time phenomena occur simultaneously. The relationship between rational streams and $\omega$-regular infinite words from formal language theory is yet another subject that deserves further study.

\section{REFERENCES}

[AS03] J.-P. Allouche and J. Shallit. Automatic sequences: theory, applications, generalizations. Cambridge University Press, 2003.

[BM77] G. Birkhoff and S. MacLane. A survey of modern algebra (4th edition). MacMillan Publishing Co., Inc., 1977.

[BR88] J. Berstel and C. Reutenauer. Rational series and their languages, volume 12 of EATCS Monographs on Theoretical Computer Science. Springer-Verlag, 1988.

[HCR06] H. Hansen, D. Costa, and J.J.M.M. Rutten. Synthesis of Mealy machines using derivatives. In Proceedings of CMCS 2006, volume 164(1) of ENTCS, pages 27-45. Elsevier Science Publishers, 2006.

[Kai80] T. Kailath. Linear systems. Prentice-Hall, 1980.

[Lah98] B.P. Lahti. Signal Processing \& Linear Systems. Oxford University Press, 1998.

[Rut05a] J.J.M.M. Rutten. Algebra, bitstreams, and circuits. In Proceedings of the Dresden Conference 2004 (AAA68), volume 16 of Contributions to General Algebra, pages 231-250. Verlag Johannes Heyn, 2005.

[Rut05b] J.J.M.M. Rutten. A coinductive calculus of streams. Mathematical Structures in Computer Science, 15:93-147, 2005.

[Rut07] J.J.M.M. Rutten. Coalgebraic foundations of linear systems. In T. Mossakowski, U. Montanari, and M. Haveraaen, editors, Proceedings of CALCO 2007, volume 4624 of LNCS, pages 425-446. SpringerVerlag, 2007.

Acknowledgments: This paper, as well as an earlier version of it, has been reviewed by anonymous referees. I am very grateful for the many constructive comments these referees have made. They have improved both the presentation of the paper and my understanding of its contents.

\section{APPENDiX A.}

A semi-ring $A=(A,+, \cdot, 0,1)$ is a set $A$ with a commutative operation of addition $c+d$; a (generally non-commutative) operation of multiplication $c \cdot d$ with $c \cdot(d+e)=(c \cdot d)+(c \cdot e)$ and $(d+e) \cdot c=(d \cdot c)+(e \cdot c)$; and with neutral elements 0 and 1 such that $c+0=c$, $1 \cdot c=c \cdot 1=c$ and $c \cdot 0=0 \cdot c=0$. If every $c \in A$ moreover has an additive inverse $-c$ (with $c+(-c)=0$ ) then $A$ is a ring. If moreover multiplication is commutative and every (non-zero) element $c \in A$ has a multiplicative inverse $c^{-1}$ (with $c \cdot c^{-1}=1$ ) then $A$ is a field. 